\title{
Hans Schaefer: The Future of Social Medicine
}

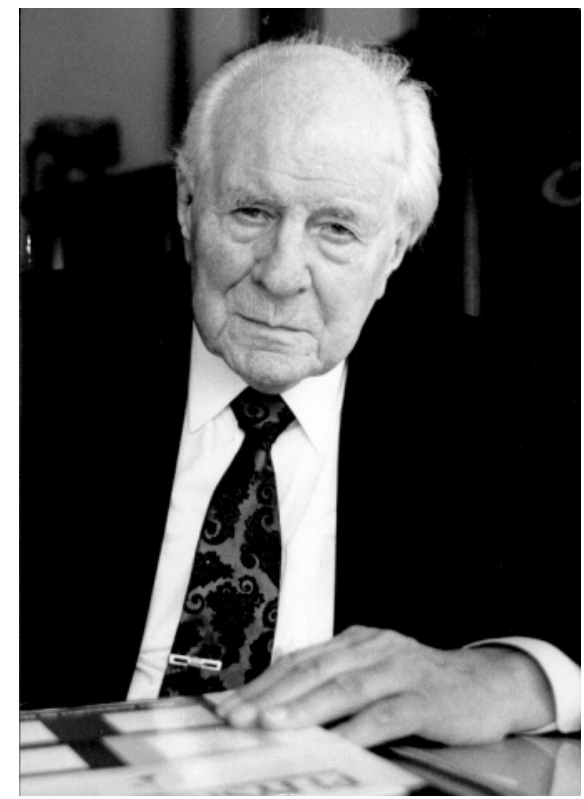

Hans Schaefer, 1998

Am 8. Februar 2003 fand eine Akademische Gedenkfeier für Prof. Dr. med. Dr. h. c. Hans Schaefer in der Alten Aula der Universität Heidelberg statt, zu der das Rektorat der Ruprecht-Karls-Universität, die Medizinische Fakultät und die Heidelberger Akademie der Wissenschaften eingeladen hatten. Die Gedenkworte und Vorträge der Feier finden Sie in diesem Heft. Hans Schaefer (13.8.1906-23.11.2000), Gründer der Deutschen Gesellschaft für Sozialmedizin und Prävention (DGSMP) im Jahr 1962, ihr langjähriger Präsident (1962-1974) und vor allem geistiger Vater der Sozialmedizin im Nachkriegsdeutschland, war im engsten
Familien- und Freundeskreis auf dem Friedhof in Heidelberg/Ziegelhausen beigesetzt worden.

Hans Schaefer hat sich nach seiner Emeritierung im Jahr 1974 nicht zurückgezogen, vielmehr beteiligte er sich bis in seine letzten Tage mit wachem Geist und abgeklärt durch reiche Lebenserfahrung kritisch, konstruktiv und weitblickend an den Entwicklungen in Gesellschaft und Wissenschaft in Vorträgen, Diskussionen und Publikationen.

An den Wissenschaftlichen Jahrestagungen der DGSMP hat Hans Schaefer regelmäßig teilgenommen, zur Freude jüngerer Sozialmediziner, die das Gespräch mit dem außergewöhnlichen Gelehrten suchten. In souveräner Voraussicht hat er auf der Marburger Tagung 1998 seinen Festvortrag als seinen letzten Auftritt in diesem Rahmen bezeichnet. Das war, für ihn und seine Umgebung spürbar, der schwindenden physischen Kraft geschuldet, ohne dass sein Interesse an der Sozialmedizin gemindert wurde, wie persönliche Gespräche und Briefwechsel in der verbleibenden Lebenszeit zeigten. In dieser Zeit habe ich Herrn Schaefer gebeten, seine Gedanken über die „Zukunft der Sozialmedizin" niederzuschreiben, zumal er 1996 in einem Manuskript zum gleichen Thema „die Sozialmedizin als Instanz der Reflexion, der Theoriebildung und vor allem der praktischen Hilfe“ bezeichnet hatte.

Hans Schaefer hat unter dem Titel „Zukunft der Sozialmedizin“ 19 Punkte ohne Kommentierung verfasst und mir 1998 zugesandt. Sie sind bisher unveröffentlicht geblieben. Ich nehme den folgenden Bericht über die Akademische Gedenkfeier zum Anlass, diese 19 Punkte der Öffentlichkeit mitzuteilen: 


\section{Zukunft der Sozialmedizin}

\section{Hans Schaefer}

1. Sozialmedizin ist eine Zwitter- oder Verbundwissenschaft: Sie vereint Medizin und Soziologie. Ihre Zukunft wird durch die Entwicklung ihrer beiden Teile bestimmt.

2. Die Soziologie ist eine primär beschreibende Wissenschaft, gleichsam eine Anatomie der Gesellschaft. Daher ihre Neigung, Gesellschaften, die sie beschreibt, an Idealen und Normen zu messen, die nur durch die zeitspezifischen Meinungen von Ideal und Norm bestimmt sind. Dadurch sind soziologische Urteile abhängig von vorgefassten Idealen, Vorstellungen einer Zeit, einer Gruppe, einer Partei.

3. Unter den Idealen, die einer Kritik der Gesellschaft (Kritik gleich kritischer Vergleich von Gesellschaften untereinander) zugrunde liegen, spielen die Vorstellungen von der Verteilung der Güter und Ressourcen immer eine hervorragende Rolle.

4. Diese Verteilungs-Ideologie beherrscht u. a. zwei fundamentale politische Prozesse: den der Verwirklichung von sozialer Gerechtigkeit und den der Inanspruchnahme durch die Individuen zur Realisierung von Ansprüchen, in erster Linie durch Solidarität. Beide sind weit unabhängiger voneinander, als man denkt.

5. Die Maß-Stäbe für solidares Verhalten schwanken zwischen den Extremen einer Gleichheitsverteilung der Ressourcen, wie sie dem Ideal der klassenlosen Gesellschaft von Marx zugrunde lag, und der Vorstellung des minimalen Supports bei maximaler Verpflichtung der Individuen, wie sie der Ideologie der Marktwirtschaft entspricht. Seltsamerweise entsteht in beiden Ideologien die Idee einer Pflicht zur Gesundheit mit der Begleitidee der Regresspflicht und der individuellen Verantwortung, freilich mit unterschiedlicher Begründung.

6. In den beiden extremen Systemen einer klassenlosen Gesellschaft und einer liberalen Marktwirtschaft wird deren Akzeptanz durch „politische Korrektheit“ bewirkt bzw. erzwungen. Dieser Begriff, in einer diktatorischen Gesellschaft selbstverständlich, beherrscht auch eine liberale Gesellschaft mit einer Praxis, die erstaunlich konform ist. Beiden Systemen ist eigentümlich, dass der Grad der „Korrektheit“, der postuliert wird, mit wachsenden ökonomischen Schwierigkeiten zunimmt. Da in unserer liberalen Gesellschaft die Schwierigkeiten, aus sehr verschiedenen Ursachen, ständig wachsen, ist eine steigende Konformität der ökonomischen Urteile auch bei uns vorauszusehen.

7. Der Sozialstaat ist ein Versuch, die Gleichgewichte der sozialen Kräfte bei maximaler Freizügigkeit der Individuen herzustellen. Es ist schon heute erkennbar, dass dieser Prozess um so mehr scheitert, je größer die Schwierigkeiten der Herstellung von Gleichgewichten werden. Das Urteil über eine gerechte Verteilung wird dann kontrovers, wenn es zu echten Einschränkungen der Bedürfnisbefriedigung kommt.

8. Insofern Sozialmedizin Teil der Medizin ist, hat sie auch damit zu tun, die Gleichgewichte auf ihre zeitbestimmte Gültigkeit zu prüfen. Sie trifft dabei auf zwei entscheidende Schwierigkeiten: Die Möglichkeiten medizinischer Therapien (Dienste) wachsen, zugleich mit ihren Kosten; die Bedürftigkeit, die immer durch Konsens festgeschrieben wird (z. B. in konzertierten Aktionen), zeigt überall die Ten- denz rascher Zunahme, durch Überalterung und „Wehleidigkeit", welch Letztere eine Umschreibung der zeitlich sich wandelnden Ansichten über Glück, Unglück, Anspruch und Recht symbolisiert.

9. Die von der Gesellschaftsordnung ins Gleichgewicht zu bringenden sozialen Prozesse verändern sich derzeit mit wahrnehmbarer Geschwindigkeit, wodurch das Gefühl der „Krise“ entsteht. Das dominierende Phänomen wachsender Ungleichheit besteht darin, dass Möglichkeiten von Leistungen und das Bedürfnis ihrer Inanspruchnahme konform wachsen, bei fast gleich verbleibender Größe der verfügbaren Mittel, mit denen sie bezahlt werden können.

10. Solidarität besteht darin, dass in einer Gesellschaft die individuellen Bedürfnisse ohne Vorbehalt anerkannt und auf der Basis der Gemeinsamkeit befriedigt werden. Diese Form der Solidarität setzt voraus, dass die Ressourcen den Bedürfnissen fließend angepasst werden.

11. Die Folgen einer solchen Solidarität der Verteilung würden in kontinuierlich wachsender Belastung der Gemeinschaft bestehen. Dieses Anwachsen der Belastung nähert sich immer einer Grenzregion, die unscharf ist und durch die ebenfalls unscharfe Empfindung der Individuen, die Belastung überschreite die Grenze der Toleranz, vorgezeichnet wird.

12. In allen Gesellschaften werden Toleranzgrenzen durch den Prozess der zeitgenössischen Ansichten über Verteilungsgerechtigkeiten bestimmt. Diese Grenzen sind immer fließend und von den gesellschaftlichen Umständen, die jeweils herrschen, bestimmt. Der Begriff der „Erträglichkeit“ einer Solidarität ist immer willkürlich und in der Regel stark durch die Historie der Verhältnisse bestimmt.

13. Diese historisch überkommenen Grenzziehungen aktualisieren sich durch zwei Entwicklungen. Zum einen wird die durch Solidarität erlaubte Belastungsgrenze verändert, zum anderen werden die Gründe der Ungleichheit, aus denen die Belastung fließt, kritisiert. Die derzeit geübte politische Praxis tut keines dieser beiden. Es wird vielmehr dekretiert, dass eine Belastungsgrenze erreicht sei, die offenbar weder durch Regelkreise marktwirtschaftlicher Art bedient werden kann noch durch Belastung der Solidarität, die also in einem der Marktwirtschaft völlig fremden Akt der Festsetzung durch staatliche Gewalt tolerabel gestaltet wird. In einer Demokratie ist aber auch dieser staatliche Gewaltakt konsensbedürftig.

14. In einer Situation, in der das Verhältnis von Bedürfnis und Bedürfnisbefriedigung sich durch steigende Möglichkeiten der Befriedigung (d.h. der therapeutischen Leistungen) zugleich mit wachsenden Ansprüchen ständig verschlechtert, werden die Konsens-Urteile über dieses Verhältnis extrem schwierig.

15. Der Konsens wird derzeit durch ein denkbar einseitiges, also kritisierbares Verfahren hergestellt, durch die „Konzertierung“, also die gemeinsame Abstimmung gleich lautender Interessen, denen ein ungebremstes finanzielles Risiko gegenübersteht, das aber als Interessenregler durch seine Indirektheit deutlich unterlegen ist: Der Anstieg der Gemeinkosten des Gesundheitswesens wird auf seine Ursachen hin schwer einsehbar.

16. Das konzertierte Interesse definiert sich dadurch, dass die Leistungsanbieter an einer Steigerung der Umsätze ebenso interessiert sind wie die Versicherungen, diese Steigerung 
aber unreflektiert vom Verhalten der Summe der Patienten erzeugt wird, ohne dass dabei ein bestimmter Handlungswille wirksam werden muss.

17. Die Aufgabe der Sozialmedizin ist es, die im Sektor Medizin einerseits, Gesellschaft andererseits herrschenden Kräfte zu beschreiben und Bilanzierungen unter Aufzeigung aller Alternativen und Konsequenzen zu planen. Die Problematik dieser Aufgabe liegt darin, dass sie notwendigerweise mit den Interessen der Gruppen in Konflikt kommt.

18. Eine konfliktfreie Strategie bleibt dennoch möglich. Es könnten sich die Bedürfnisse ändern lassen, durch Krankheitsverhütung („Salutogenese“), es könnte sich ergeben, dass auch medizinische Leistungen inkorrekt sind, z. B. durch die Frage entscheidbar sind, ob die Medizin überall das Geld wert ist, das sie kostet:
19. In einer Zeit wie der unsrigen, in der alle egoistischen Lebensziele immer stärker betont werden, ist der soziale Friede sicher nicht mehr allein mit der Berufung auf das Prinzip der Solidarität zu wahren. Solidarverhalten orientiert sich immer stärker an der Einhaltung sachbezogener Kriterien wie einer Kritik der Kostenentwicklung. Dass auch die Inanspruchnahme gleiche Bedeutung hat, ist deshalb sehr viel schwerer zu vermitteln, weil die Kritik der „Bedürfnisse“ dem Lebensgefühl unserer Zeit zuwiderläuft. Es bedarf besonderer Methoden, die Einsicht zu vermitteln, dass individuelle Interessen auch dadurch berührt werden, dass eine Gesellschaft individuelle Bedürfnisse kritiklos befriedigt. Gerade dieser Punkt beweist wieder, wie stark alle sozialen Urteile vom Zeitgeist abhängen. 